Research Article

\title{
Electrical Signal Guided Ibuprofen Release from Electrodeposited Chitosan Hydrogel
}

\author{
Youyu Liu, Kun Yan, Guoxia Jiang, Yuan Xiong, Yumin Du, and Xiaowen Shi
}

School of Resource and Environmental Science and Hubei Biomass-Resource, Chemistry and Environmental Biotechnology Key Laboratory, Wuhan University, Wuhan 430079, China

Correspondence should be addressed to Xiaowen Shi; shixwwhu@163.com

Received 5 March 2014; Accepted 2 August 2014; Published 19 August 2014

Academic Editor: Qijin Zhang

Copyright (c) 2014 Youyu Liu et al. This is an open access article distributed under the Creative Commons Attribution License, which permits unrestricted use, distribution, and reproduction in any medium, provided the original work is properly cited.

Electrical signal guided drug release from conductive surface provides a simple and straightforward way for advanced drug delivery. In this study, we investigated the ibuprofen release from electrodeposited chitosan hydrogel by applying electrical signals. Specifically, chitosan hydrogel was electrodeposited on titanium plate and used as a matrix for ibuprofen load and release. The release of ibuprofen from the chitosan hydrogel on titanium plate was $\mathrm{pH}$ sensitive. By applying a positive or negative electrical potential, the release rate of ibuprofen from the electrodeposited chitosan can be facilely controlled. Thus, coupling chitosan electrodeposition and electrical signal control spurs new possibilities for biopolymeric coating and drug elution on conductive implants.

\section{Introduction}

External stimuli triggered drug release alters the traditional way of drug delivery and causes substantial attention and interests [1]. Generally, on demand release happens when external stimuli such as light [2], magnetic field [3,4], heat [5], or $\mathrm{pH}[6]$ were applied to the release matrix. Electrical signal is simple to use and has the advantage of easy combination to remote control, and, thus, it has been used in embedded drug delivery chips $[7,8]$. Recently, using electrical signal to control the drug release from biopolymers prompts even more opportunities for biomedicine and advanced therapy.

Chitosan is an aminopolysaccharide derived from chitin, which can be found in the shells of crabs and shrimps [9]. Due to its intriguing biocompatibility, biodegradability, and nontoxicity, chitosan has been studied extensively as drug carrier in the field of pharmaceutics and biomedicine [10-16]. Previously, we showed that chitosan could be electrodeposited on electrodes as a hydrogel by enlisting its $\mathrm{pH}$ responsive and film forming properties [17, 18]. The electrodeposition process includes the neutralization of chitosan chains by locally generated $\mathrm{pH}$ gradient and the sol-gel transition of chitosan in situ $[19,20]$. Strong noncovalent bonds such as hydrogen bonds and hydrophobic interactions are responsible for the generation of chitosan hydrogel. Various biocomponents such as proteins [21-23], cells [24, 25], antibiotics [26], and nanocomponents [27] can be entrapped in the porous network of the deposited chitosan hydrogel. The above work demonstrates the capability of electrodeposited chitosan as drug reservoir and the potential for controlled drug release.

In this work, we demonstrate the controlled drug release from electrodeposited chitosan by applying electrical signals. The manipulation of electrical signals causes the on-site generation of chitosan hydrogel and sequentially controlled drug release from the hydrogel. It couples the advantage of chitosan hydrogel as preferable drug carrier and the straightforwardness of electrical signals. The procedure was illustrated in Scheme 1. Firstly, chitosan was electrodeposited on titanium plate by biasing a negative potential to generate a hydrogel. Then the titanium plate with electrodeposited chitosan hydrogel was immersed in ibuprofen solution $(\mathrm{pH}$ 10) for drug load. The profile of ibuprofen release from the chitosan hydrogel was adjusted by applying electrical potentials. Electrochemical reactions on the titanium plate caused by the negative or positive potential changed the local $\mathrm{pH}$ of the chitosan hydrogel, thus varying the ibuprofen release rate. We believe that this easy and benign drug load 


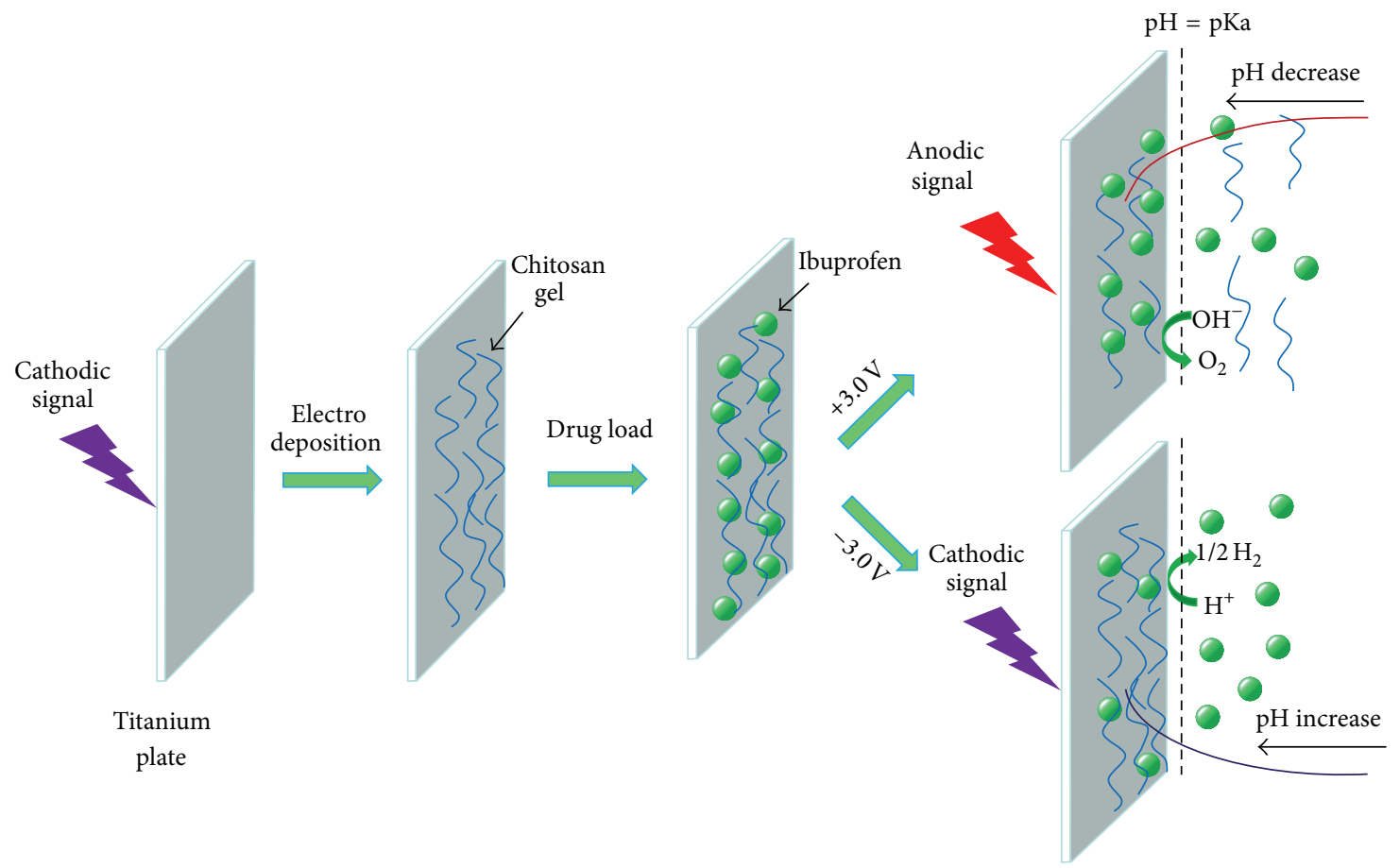

Scheme 1: Illustration of electrical signals guided ibuprofen release from electrodeposited chitosan hydrogel.

and release procedure should have potential applications in smart drug release from conductive implant or lab on a chip.

\section{Experimental}

2.1. Materials. Chitosan with medium molecular weight and a degree of deacetylation (DD) of $85 \%$ was purchased from Sigma. Ibuprofen was purchased from Shanghai Yuan-Ji Chemical Reagent Co., Ltd. Titanium plates with a thickness of $100 \mu \mathrm{m}$ were purchased from Baoji Titanium Company, Shanxi. Other chemicals were of analytical grade and were purchased from Sinopharm Chemical Reagent Co. without further purification.

\subsection{Electrodeposition of Chitosan Hydrogel on Titanium Plates.} Electrodeposition of chitosan hydrogel on titanium plates was carried out by using a conventional two-electrode system as described previously [26]. The working electrode was a titanium plate with a size of $1.5 \times 3.5 \mathrm{~cm}^{2}$ and the reference electrode was a platinum wire. All the electrodes were sonicated consecutively before use in the bath of acetone, ethanol, and water for 5 min each. Chitosan solution (1\%, wt) was prepared by mixing chitosan flakes with distilled water followed by adjusting the $\mathrm{pH}$ to 3 by adding $\mathrm{HCl}$ solution. Undissolved part was removed by a $0.45 \mu \mathrm{m}$ syringe filter unit. The $\mathrm{pH}$ of chitosan solution was adjusted to $\sim 5$ by using $1 \mathrm{M} \mathrm{NaOH}$. Before deposition, $\mathrm{H}_{2} \mathrm{O}_{2}$ and $\mathrm{NaCl}$ were added to the chitosan solution to a final concentration of $20 \mathrm{mM}$ for $\mathrm{H}_{2} \mathrm{O}_{2}$ and $43 \mathrm{mM}$ for $\mathrm{NaCl}$ [28]. The electrodes were partially dipped into the chitosan solution and the distance between the electrodes and the immersion depth were both kept at
$2 \mathrm{~cm}$. Then the electrodes were connected to a power supply (CHI 624D electrochemistry workstation, Shanghai, China) and applied a constant voltage of $-3.0 \mathrm{~V}$ for $30 \mathrm{~min}$. After that, the titanium plate with electrodeposited chitosan hydrogel was removed from the system and rinsed briefly with distilled water for further usage.

2.3. Drug Load on Titanium Plate. Various concentrations of ibuprofen solution were prepared by dissolving ibuprofen flakes in glycine/sodium hydroxide buffer and the $\mathrm{pH}$ was adjusted to 10 by adding $1 \mathrm{M} \mathrm{NaOH}$. The titanium plate with deposited chitosan hydrogel was immersed in ibuprofen solution for $1 \mathrm{~h}$. Then the titanium plate with chitosan hydrogel was removed from the ibuprofen solution and rinsed carefully with distilled water. The amount of ibuprofen loaded in chitosan hydrogel was measured by UV-Vis spectroscopy at $264 \mathrm{~nm}$ and determined as the percentage of ibuprofen to the total weight of drug loaded chitosan gel.

2.4. Characterization. The morphology of the chitosan hydrogel with loaded ibuprofen was examined by scanning electron microscopy (SEM). The dried chitosan-ibuprofen coating on the titanium plate was sputtered with gold and the morphology of the film was observed at an acceleration voltage of $5 \mathrm{kV}$. The infrared spectrum of the chitosan-ibuprofen coating was collected by Fourier transform infrared spectroscopy (FT-IR, Nicolet Corporation 5700) using KBr pellets method. The X-ray diffraction (XRD) patterns were obtained on a SHIMADZU XRD-6000 diffractometer $(\mathrm{Cu} \mathrm{K} \alpha$ radiation) with a multipurpose sample holder for nondestructive analysis. 


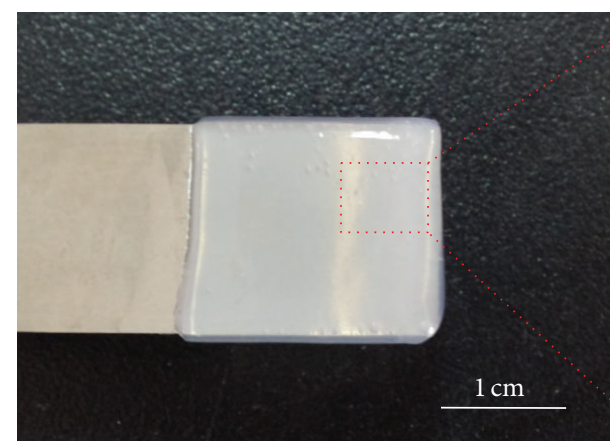

(a)

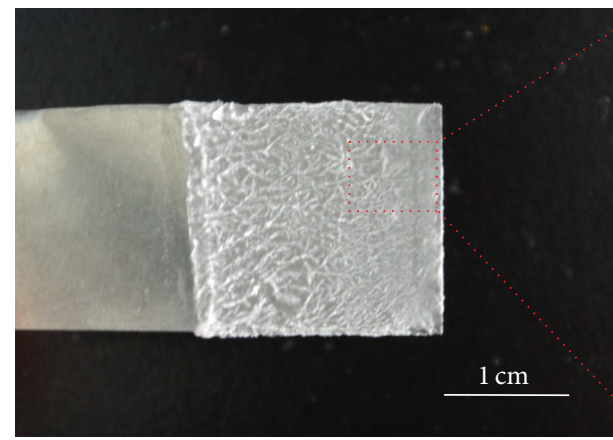

(c)

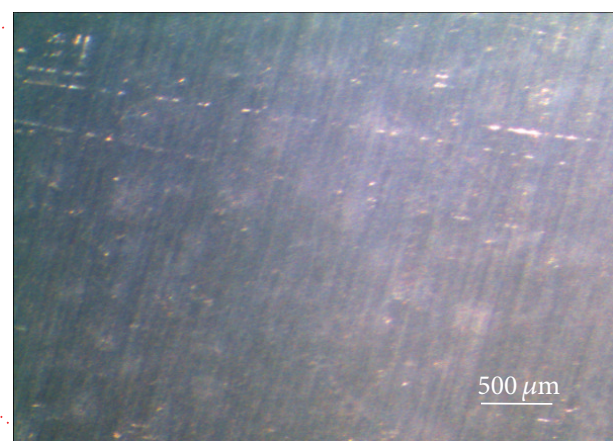

(b)

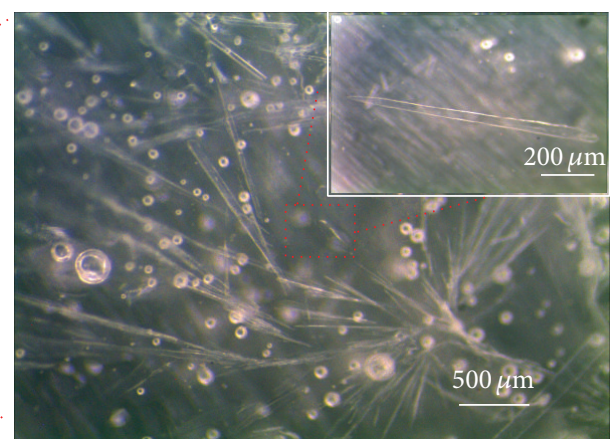

(d)

FIGURE 1: Photographs of the chitosan-ibuprofen composite hydrogel on titanium plate. Wet (a) and dried (c) chitosan-ibuprofen composite hydrogel and its corresponding magnified photographs (b) and (d). The insert of (d) shows the enlarged ibuprofen needle-like crystal.

2.5. Electrical and $\mathrm{pH}$ Controlled Drug Release. The $\mathrm{pH}$ responsive ibuprofen release from electrodeposited chitosan hydrogel was monitored by immersing the titanium plate with deposited chitosan in $20 \mathrm{~mL}$ buffer solutions with different $\mathrm{pH}$ values ( $\mathrm{pH} 4,7$, and 10). At predetermined intervals, the released ibuprofen was measured by UV absorbance at $264 \mathrm{~nm}$. Each assay was performed in triplicate and reported as the average with standard deviation.

The electrical signal controlled drug release was carried out by immersing titanium plates with chitosan-ibuprofen composite hydrogel in $\mathrm{NaCl}$ solution $(0.9 \%$, w/v). The release of ibuprofen was controlled by applying a voltage to the titanium plate (voltage typically varied between $-3 \mathrm{~V}$ and $+3 \mathrm{~V}$ ) with a platinum wire working as counter electrode. The elution of ibuprofen responding to applied voltage and time was measured by UV absorbance at $264 \mathrm{~nm}$.

\section{Results and Discussion}

We first deposited chitosan hydrogel on a titanium plate under a constant current of $0.5 \mathrm{~mA}$ for $30 \mathrm{~min}$ and then immersed the hydrogel in ibuprofen solution $(1 \%, \mathrm{wt})$ for $1 \mathrm{~h}$. Figure 1 shows the photographs of the composite chitosanibuprofen hydrogel. The hydrogel is transparent and uniform with a smooth surface (Figures 1(a) and 1(b)). The size of the hydrogel is $1.5 \times 2.0 \mathrm{~cm}$, which is corresponding to the area dipped in chitosan solution during electrodeposition process. The thickness is estimated to be $1 \mathrm{~mm}$. When the hydrogel was air-dried, irregular white textures appeared on the titanium plate (Figure 1(c)). The texture was further observed by a photomicroscope. Crystals of ibuprofen with needle shape are well dispersed in chitosan film (Figure 1(d), the insert of Figure 1(d) shows one crystal with $500 \mu \mathrm{m}$ length and $30 \mu \mathrm{m}$ width). The results in Figure 1 preliminarily suggest that ibuprofen can be loaded in the deposited chitosan hydrogel.

The chitosan-ibuprofen composite film was further characterized by SEM. Figure 2 shows the SEM images of the surface of chitosan-ibuprofen composite film. Chitosan film without ibuprofen displays a relatively smooth surface with micropores of $\sim 0.6 \mu \mathrm{m}$ (Figure $2(\mathrm{a})$ ). When the chitosan hydrogel was immersed in $0.2 \%$ of ibuprofen solution for $1 \mathrm{~h}$, the surface of the chitosan film became rough. The fibril structure on the surface was attributed to the crystallization of ibuprofen in chitosan membrane (Figure 2(b)). Figures 3(b) and 4(b) exhibit the surface morphology of chitosanibuprofen in $0.6 \%$ and $1.0 \%$ of ibuprofen solution, respectively. Obvious crystal structure can be observed with the increased concentration of ibuprofen. It is interesting to note that ibuprofen crystal shows well-oriented layer structure, as revealed by inset images in Figures 2(c) and 2(d). It has been reported that the morphology of the recrystallized ibuprofen depends on the crystallization solvents and additives. The crystal forms of ibuprofen were found as $\{100\},\{002\}$, and $\{011\}$ [29]. The carboxyl groups of ibuprofen project out from the face (100) and can form hydrogen bonding with hydroxyl groups on chitosan, thus reducing the growth rate on this face. The growth on (011) face leads to large platelet structure as observed in SEM. 


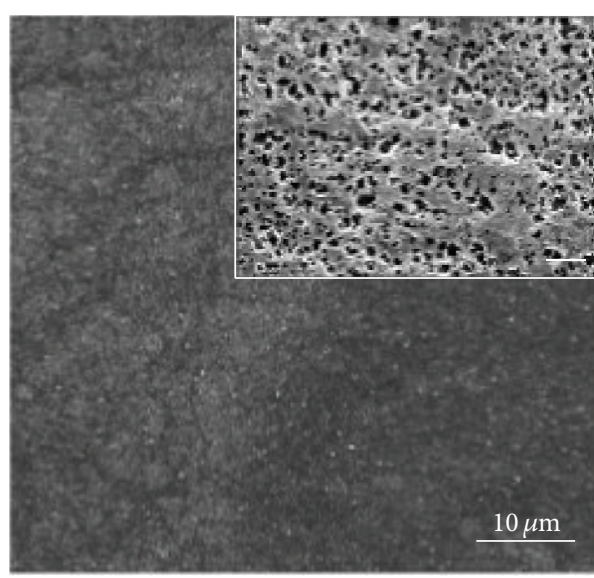

(a)

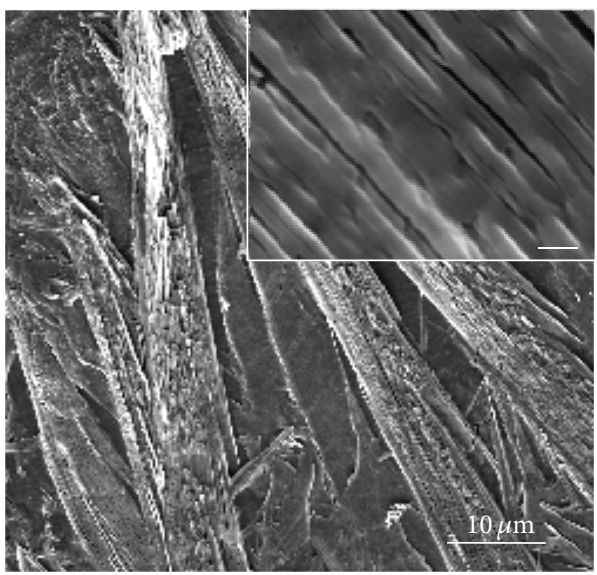

(c)

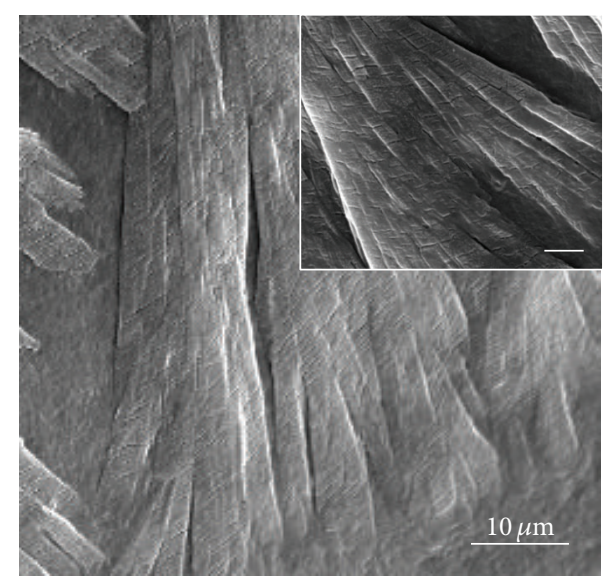

(b)

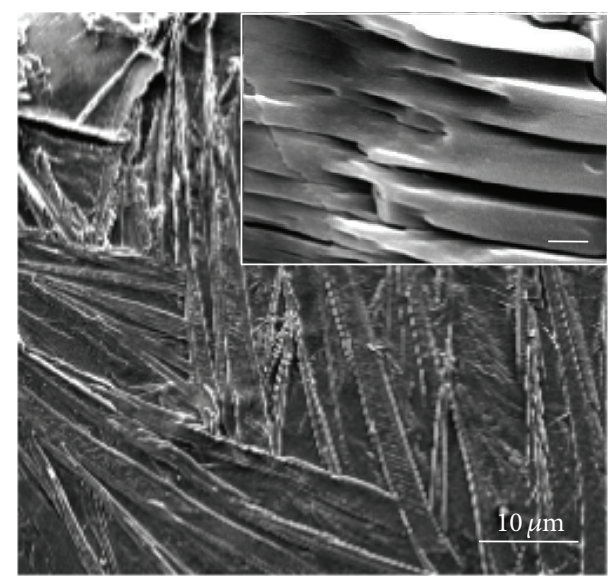

(d)

FIGURE 2: SEM images of the surface of the chitosan-ibuprofen composite hydrogel on titanium plate. Pure chitosan hydrogel (a); chitosan hydrogel with $0.2 \%$ ibuprofen (b); chitosan hydrogel with $0.6 \%$ ibuprofen (c); and chitosan hydrogel with $1.0 \%$ ibuprofen (d). The insert of each SEM image shows high magnified images (the scale bar was $2 \mu \mathrm{m}$ ).

The chitosan-ibuprofen composite film was peeled off from the titanium plate and analyzed by FT-IR (Figure 3 ). The spectra were compared to that of pure ibuprofen and chitosan. Ibuprofen exhibits characteristic peaks at $2955 \mathrm{~cm}^{-1}$ and $1719 \mathrm{~cm}^{-1}$, which can be attributed to $\mathrm{O}-\mathrm{H}$ stretching and carbonyl stretching, respectively. Due to the presence of benzene ring, the strong peak at $779 \mathrm{~cm}^{-1}$ represents the aromatic $\mathrm{C}-\mathrm{H}$ stretching [30]. The spectrum of chitosan shows absorption bands at $1650 \mathrm{~cm}^{-1}$ and $1553 \mathrm{~cm}^{-1}$, corresponding to carbonyl stretching and amide II absorption, respectively. The FT-IR spectra of chitosan-ibuprofen composite films show the peaks at $779 \mathrm{~cm}^{-1}$ and $2955 \mathrm{~cm}^{-1}$, providing evidence that ibuprofen exists in the composite film. However, there is no peak showed at $1719 \mathrm{~cm}^{-1}$, suggesting that the carbonyl group might be ionized in the membrane.

The X-ray diffraction patterns of chitosan-ibuprofen composite films are provided in Figure 4. The sharp and intense peaks at $12.1^{\circ}, 16.6^{\circ}, 17.7^{\circ}$, and $22.2^{\circ}$ suggest that ibuprofen is highly crystallized [31]. The wide crystalline peak at $19.8^{\circ}$ in chitosan indicates its partial crystallization feature. In the chitosan-ibuprofen composite films, the crystalline peaks of ibuprofen remain and the intensity is enhanced with the increased amount of loaded ibuprofen, suggesting that the ibuprofen stays in crystalline state in the composite film.

To determine the amount of ibuprofen incorporated in chitosan-ibuprofen films, the ibuprofen in the film was extracted by immersing the film in ethanol for $1 \mathrm{~h}$ and its concentration was determined by UV-Vis spectroscopy at $264 \mathrm{~nm}$. As shown in Figure 5, the amount of loaded ibuprofen in chitosan hydrogel increases proportionally to the concentration of ibuprofen. Due to the limited solubility of ibuprofen in water phase, the highest concentration of ibuprofen tested in this study was $1 \%$. In this case, the load percentage of ibuprofen reaches $39.04 \%$. This relatively large carrying amount of ibuprofen indicates remarkable drug load capacity of the electrodeposited chitosan hydrogel.

Next we investigated the influence of $\mathrm{pH}$ in release medium on the release profile of ibuprofen. Figure 6 shows the profiles of ibuprofen release under various $\mathrm{pH}$ values. It is obvious that the release rate in neutral buffer $(\mathrm{pH}=7)$ was noticeably higher than that in acidic solution $(\mathrm{pH}=4)$ but lower than that in base condition $(\mathrm{pH}=10)$. As ibuprofen is 


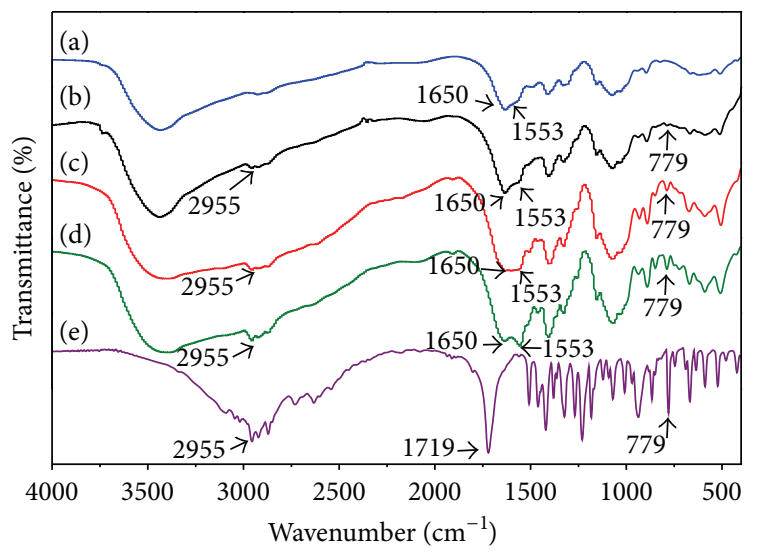

FIGURE 3: FT-IR spectra of the chitosan hydrogels with different concentrations of ibuprofen. Chitosan (a); chitosan with $0.2 \%$ ibuprofen (b); chitosan with $0.6 \%$ ibuprofen (c); chitosan with $1.0 \%$ ibuprofen (e); and ibuprofen (d).

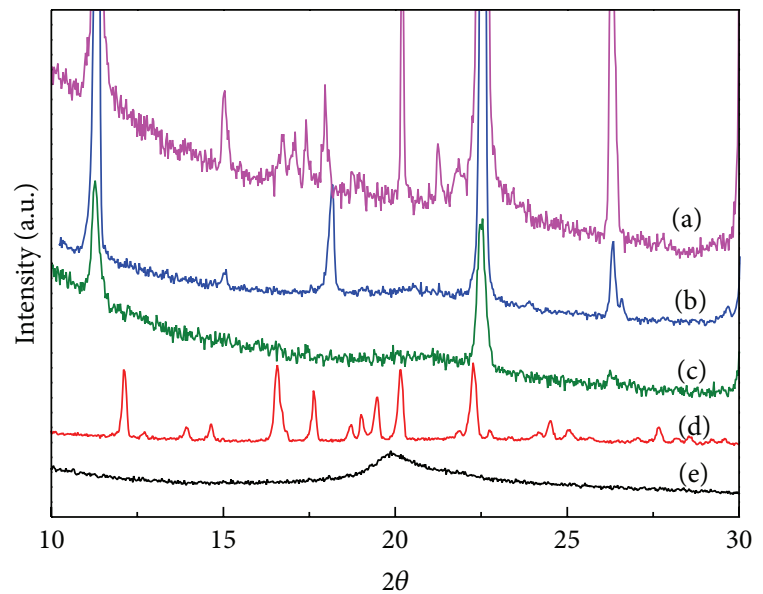

FIGURE 4: XRD pattern of chitosan hydrogel with different concentrations of ibuprofen. Chitosan with $1.0 \%$ ibuprofen (a); chitosan with $0.6 \%$ ibuprofen (b); chitosan with $0.2 \%$ ibuprofen (c); ibuprofen (d); and chitosan (e).

a weak acidic drug, the elution of ibuprofen from chitosan is $\mathrm{pH}$ dependent. It has a small solubility in acid and can be easily dissolved at high $\mathrm{pH}$. At $\mathrm{pH} 4$, less than $30 \%$ of ibuprofen was released in $24 \mathrm{~h}$, while this value increased with the increase of $\mathrm{pH}$ in the release medium. At $\mathrm{pH} 10$, ibuprofen was released to $64 \%$ in the first $2 \mathrm{~h}$ and approximately to $78 \%$ in $12 \mathrm{~h}$. The release profiles of ibuprofen indicate the important role of $\mathrm{pH}$ in the release of ibuprofen.

Inspired by the $\mathrm{pH}$ mediated ibuprofen release, we tested the possibility of ibuprofen release controlled by using electrical signals. In general, the electrochemical reaction induced by applied potential will result in $\mathrm{pH}$ gradient near the electrodes. The electrolysis of water induces a local $\mathrm{pH}$ increase at the cathode and a local $\mathrm{pH}$ decrease at the anode $[32,33]$. The titanium plate with ibuprofen loaded chitosan hydrogel was immersed in $0.9 \% \mathrm{NaCl}$ and external potential was applied $(-3 \mathrm{~V}, 0 \mathrm{~V},+3 \mathrm{~V})$. As shown in Figure 7(a), ibuprofen release

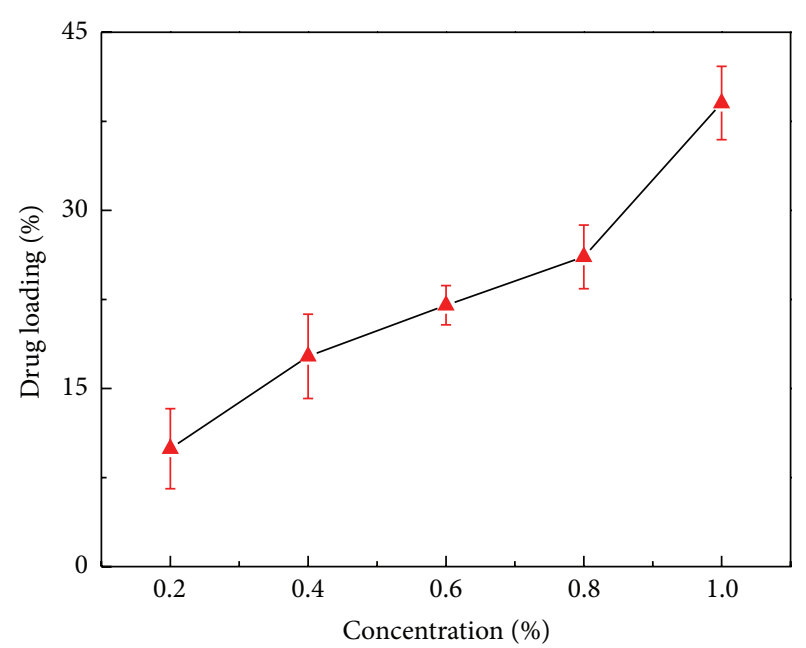

FIGURE 5: Ibuprofen loading in chitosan hydrogel by immersing titanium plates in various concentrations of ibuprofen.

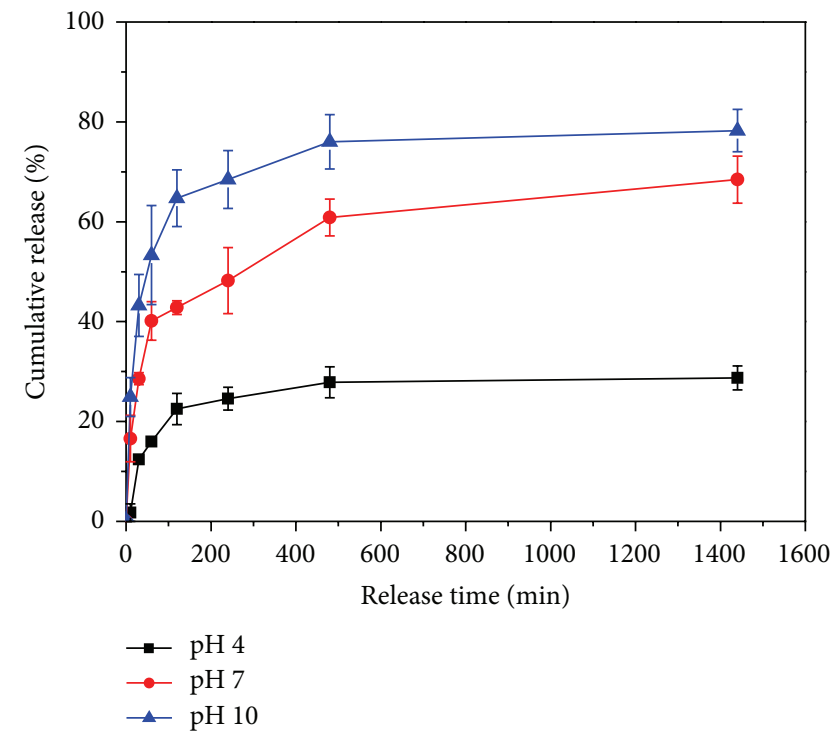

FIGURE 6: Cumulative release profiles of ibuprofen from chitosanibuprofen hydrogels under different $\mathrm{pH}$ conditions.

from the composite hydrogel depends on the voltage applied for drug release. In the first $10 \mathrm{~min}, 14 \%$ of ibuprofen was released from the titanium plate at $+3.0 \mathrm{~V}$, while only $0.8 \%$ of ibuprofen was released at $-3.0 \mathrm{~V}$. This discrepancy can be explained by the different electrochemically generated local $\mathrm{pH}$ values adjacent to titanium plate that modifies the elution behavior of ibuprofen from the chitosan hydrogel. By biasing a negative potential $(-3.0 \mathrm{~V})$, the consumption of $\mathrm{H}^{+}$will increase the $\mathrm{pH}$ near the titanium plate, thus shrinking the chitosan hydrogel on its surface. On the other hand, the increased $\mathrm{pH}$ also facilitates the dissolution of ibuprofen. The amount of ibuprofen release reached a plateau of $66 \%$ at $-3.0 \mathrm{~V}$, while the total cumulative release was merely $31 \%$ at $+3.0 \mathrm{~V}$. Figure 7(b) and Figure 7(c) show the SEM images of chitosan hydrogels by applying $-3.0 \mathrm{~V}$ and 


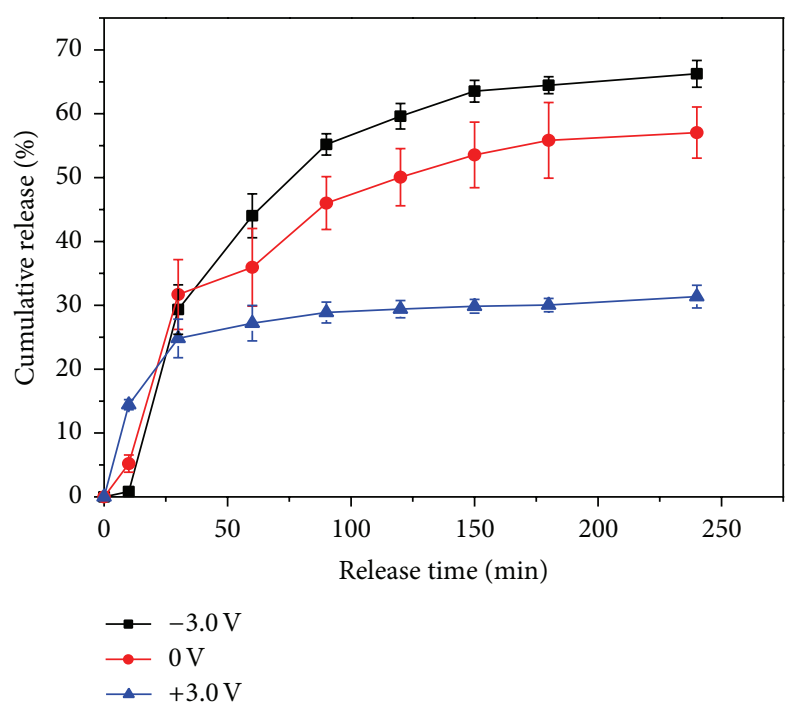

(a)

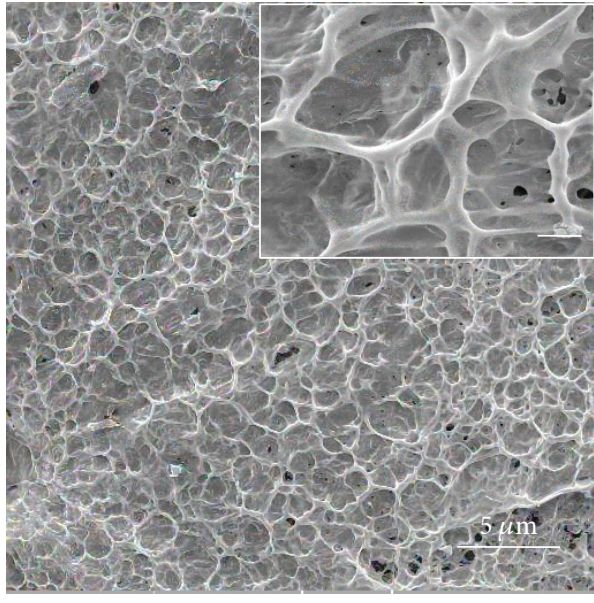

(b)

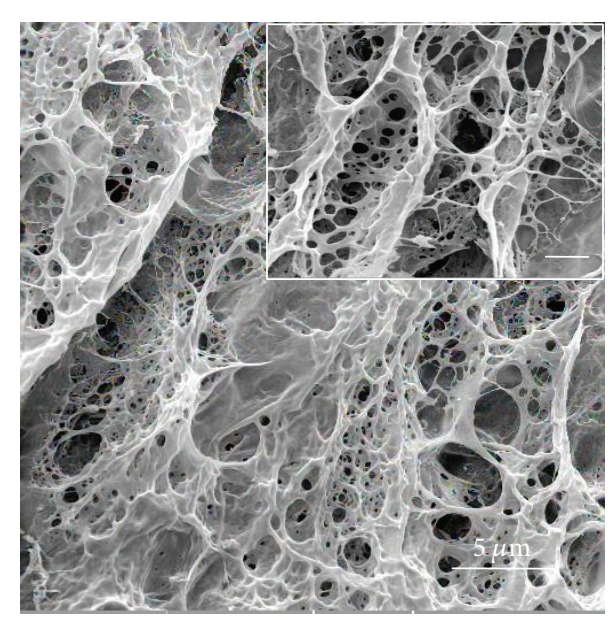

(c)

FIGURE 7: Cumulative release of ibuprofen from chitosan-ibuprofen hydrogels by applying external potentials (a) and SEM images of the chitosan hydrogel after ibuprofen release by applying $-3.0 \mathrm{~V}$ (b) and $+3.0 \mathrm{~V}$ (c). The insert of SEM image shows high magnified images (the scale bar was $1 \mu \mathrm{m})$.

$+3.0 \mathrm{~V}$, respectively. No large ibuprofen crystals can be seen, indicating the release of ibuprofen. The hydrogel under $+3.0 \mathrm{~V}$ reveals a more porous morphology than the hydrogel under $-3.0 \mathrm{~V}$, which is in agreement with the different $\mathrm{pH}$ environments at different voltages. The results in Figure 7 demonstrate that the flexible ibuprofen release profile can be mediated by applying varying potentials.

\section{Conclusions}

In this report, we electrodeposited chitosan on titanium plate and ibuprofen was loaded efficiently by a simple immersion method. The elution of ibuprofen is $\mathrm{pH}$ dependent. As a proof of concept study, the release profile of ibuprofen can be controlled by biasing negative or positive potentials, which is dramatically different from the situation of no voltage applied. The results provided in this work suggest new possibilities for building functional biopolymeric coat on conductive implant and controlling drug release by external electrical signals.

\section{Conflict of Interests}

The authors declare no competing financial interest.

\section{Authors' Contribution}

Youyu Liu and Kun Yan contributed equally to this work.

\section{Acknowledgments}

This work was financially supported by the National Natural Science Foundation of China (Grant nos. 51373124 and 21007049), Program for New Century Excellent Talents in University (NECT-10-0618), and Natural Science Foundation 
of Hubei Province (2011CDB268). The authors thank the Large-Scale Instrument and Equipment Sharing Foundation of Wuhan University for the support.

\section{References}

[1] Y. Qiu and K. Park, "Environment-sensitive hydrogels for drug delivery," Advanced Drug Delivery Reviews, vol. 64, pp. 49-60, 2012.

[2] J. Lu, E. Choi, F. Tamanoi, and J. I. Zink, "Light-activated nanoimpeller-controlled drug release in cancer cells," Small, vol. 4, no. 4, pp. 421-426, 2008.

[3] T. Liu, S. Hu, K. Liu, D. Liu, and S. Chen, "Preparation and characterization of smart magnetic hydrogels and its use for drug release," Journal of Magnetism and Magnetic Materials, vol. 304, no. 1, pp. e397-e399, 2006.

[4] N. S. Satarkar and J. Z. Hilt, "Magnetic hydrogel nanocomposites for remote controlled pulsatile drug release," Journal of Controlled Release, vol. 130, no. 3, pp. 246-251, 2008.

[5] X.-Z. Zhang, D.-Q. Wu, and C.-C. Chu, "Synthesis, characterization and controlled drug release of thermosensitive IPNPNIPAAm hydrogels," Biomaterials, vol. 25, no. 17, pp. 37933805, 2004.

[6] S. Chen, Y. Wu, F. Mi, Y. Lin, L. Yu, and H. Sung, "A novel pHsensitive hydrogel composed of N,O-carboxymethyl chitosan and alginate cross-linked by genipin for protein drug delivery," Journal of Controlled Release, vol. 96, no. 2, pp. 285-300, 2004.

[7] R. Farra, N. F. Sheppard Jr., L. McCabe et al., "First-in-human testing of a wirelessly controlled drug delivery microchip," Science Translational Medicine, vol. 4, no. 122, 2012.

[8] J. H. Prescott, S. Lipka, S. Baldwin et al., "Chronic, programmed polypeptide delivery from an implanted, multireservoir microchip device," Nature Biotechnology, vol. 24, no. 4, pp. 437-438, 2006.

[9] M. Rinaudo, "Chitin and chitosan: properties and applications," Progress in Polymer Science, vol. 31, no. 7, pp. 603-632, 2006.

[10] H. Onishi, Y. Saito, M. Sasatsu, and Y. MacHida, "Kinetic analysis of in vitro and in vivo release of prednisolone from the conjugate of glycol-chitosan and succinyl-prednisolone," International Journal of Pharmaceutics, vol. 410, no. 1-2, pp. 1722, 2011.

[11] A. di Martino, M. Sittinger, and M. V. Risbud, "Chitosan: a versatile biopolymer for orthopaedic tissue-engineering," Biomaterials, vol. 26, no. 30, pp. 5983-5990, 2005.

[12] Q. Wang, N. Zhang, X. Hu, J. Yang, and Y. Du, "Chitosan/ polyethylene glycol blend fibers and their properties for drug controlled release," Journal of Biomedical Materials Research A, vol. 85, no. 4, pp. 881-887, 2008.

[13] Q. Wang, N. Zhang, X. Hu, J. Yang, and Y. Du, "Chitosan/starch fibers and their properties for drug controlled release," European Journal of Pharmaceutics and Biopharmaceutics, vol. 66, no. 3, pp. 398-404, 2007.

[14] T. Oosegi, H. Onishi, and Y. Machida, "Gastrointestinal distribution and absorption behavior of Eudragit-coated chitosanprednisolone conjugate microspheres in rats with TNBSinduced colitis," International Journal of Pharmaceutics, vol. 348, no. 1-2, pp. 80-88, 2008.

[15] D. Narayanan, A. Anitha, R. Jayakumar, and K. P. Chennazhi, "PTH 1-34 loaded thiolated chitosan nanoparticles for osteoporosis: oral bioavailability and anabolic effect on primary osteoblast cells," Journal of Biomedical Nanotechnology, vol. 10, pp. 166-178, 2014.

[16] A. Anitha, S. Uthaman, S. V. Nair, R. Jayakumar, and V. Lakshmanan, "Enhanced delivery system of flutamide loaded chitosan-dextran sulphate nanoparticles for prostate cancer," Journal of Biomedical Nanotechnology, vol. 9, no. 3, pp. 335-347, 2013.

[17] X.-W. Shi, L. Qiu, Z. Nie, L. Xiao, G. F. Payne, and Y. Du, "Protein addressing on patterned microchip by coupling chitosan electrodeposition and "electro-click" chemistry", Biofabrication, vol. 5, no. 4, Article ID 041001, 2013.

[18] X. Wei, G. F. Payne, X. Shi, and Y. Du, "Electrodeposition of a biopolymeric hydrogel in track-etched micropores," Soft Matter, vol. 9, no. 7, pp. 2131-2135, 2013.

[19] Y. Liu, B. Zhang, K. M. Gray et al., "Electrodeposition of a weak polyelectrolyte hydrogel: remarkable effects of salt on kinetics, structure and properties," Soft Matter, vol. 9, no. 9, pp. 27032710, 2013.

[20] Y. Cheng, X. Luo, J. Betz et al., "In situ quantitative visualization and characterization of chitosan electrodeposition with paired sidewall electrodes," Soft Matter, vol. 6, no. 14, pp. 3177-3183, 2010.

[21] Y. Wang, Y. Liu, Y. Cheng et al., "Coupling electrodeposition with layer-by-layer assembly to address proteins within microfluidic channels," Advanced Materials, vol. 23, no. 48, pp. 5817-5821, 2011.

[22] K. M. Gray, B. D. Liba, Y. Wang et al., "Electrodeposition of a biopolymeric hydrogel: potential for one-step protein electroaddressing," Biomacromolecules, vol. 13, no. 4, pp. 11811189, 2012.

[23] X.-W. Shi, X. Yang, K. J. Gaskell et al., "Reagentless protein assembly triggered by localized electrical signals," Advanced Materials, vol. 21, no. 9, pp. 984-988, 2009.

[24] W. Ghach, M. Etienne, P. Billard, F. P. A. Jorand, and A. Walcarius, "Electrochemically assisted bacteria encapsulation in thin hybrid sol-gel films," Journal of Materials Chemistry B, vol. 1, no. 7, pp. 1052-1059, 2013.

[25] C. Hao, L. Ding, X. Zhang, and H. Ju, "Biocompatible conductive architecture of carbon nanofiber-doped chitosan prepared with controllable electrodeposition for cytosensing," Analytical Chemistry, vol. 79, no. 12, pp. 4442-4447, 2007.

[26] X. Shi, H. Wu, Y. Li, X. Wei, and Y. Du, "Electrical signals guided entrapment and controlled release of antibiotics on titanium surface," Journal of Biomedical Materials Research A, vol. 101, no. 5, pp. 1373-1378, 2013.

[27] G. F. Payne and S. R. Raghavan, "Chitosan: a soft interconnect for hierarchical assembly of nano-scale components," Soft Matter, vol. 3, no. 5, pp. 521-527, 2007.

[28] K. Yan, F. Ding, W. E. Bentley, H. Deng, Y. Du, G. F. Payne et al., "Coding for hydrogel organization through signal guided selfassembly," Soft Matter, vol. 10, pp. 465-469, 2014.

[29] H. Cano, N. Gabas, and J. P. Canselier, "Experimental study on the ibuprofen crystal growth morphology in solution," Journal of Crystal Growth, vol. 224, no. 3-4, pp. 335-341, 2001.

[30] I. A. Sogias, A. C. Williams, and V. V. Khutoryanskiy, "Chitosanbased mucoadhesive tablets for oral delivery of ibuprofen," International Journal of Pharmaceutics, vol. 436, no. 1-2, pp. 602-610, 2012.

[31] S. Mallick, S. Pattnaik, K. Swain et al., "Formation of physically stable amorphous phase of ibuprofen by solid state milling with kaolin," European Journal of Pharmaceutics and Biopharmaceutics, vol. 68, no. 2, pp. 346-351, 2008. 
[32] S. Murdan, "Electro-responsive drug delivery from hydrogels," Journal of Controlled Release, vol. 92, no. 1-2, pp. 1-17, 2003.

[33] S. H. Yuk, S. H. Cho, and H. B. Lee, "Electric current-sensitive drug delivery systems using sodium alginate/polyacrylic acid composites," Pharmaceutical Research, vol. 9, no. 7, pp. 955-957, 1992. 

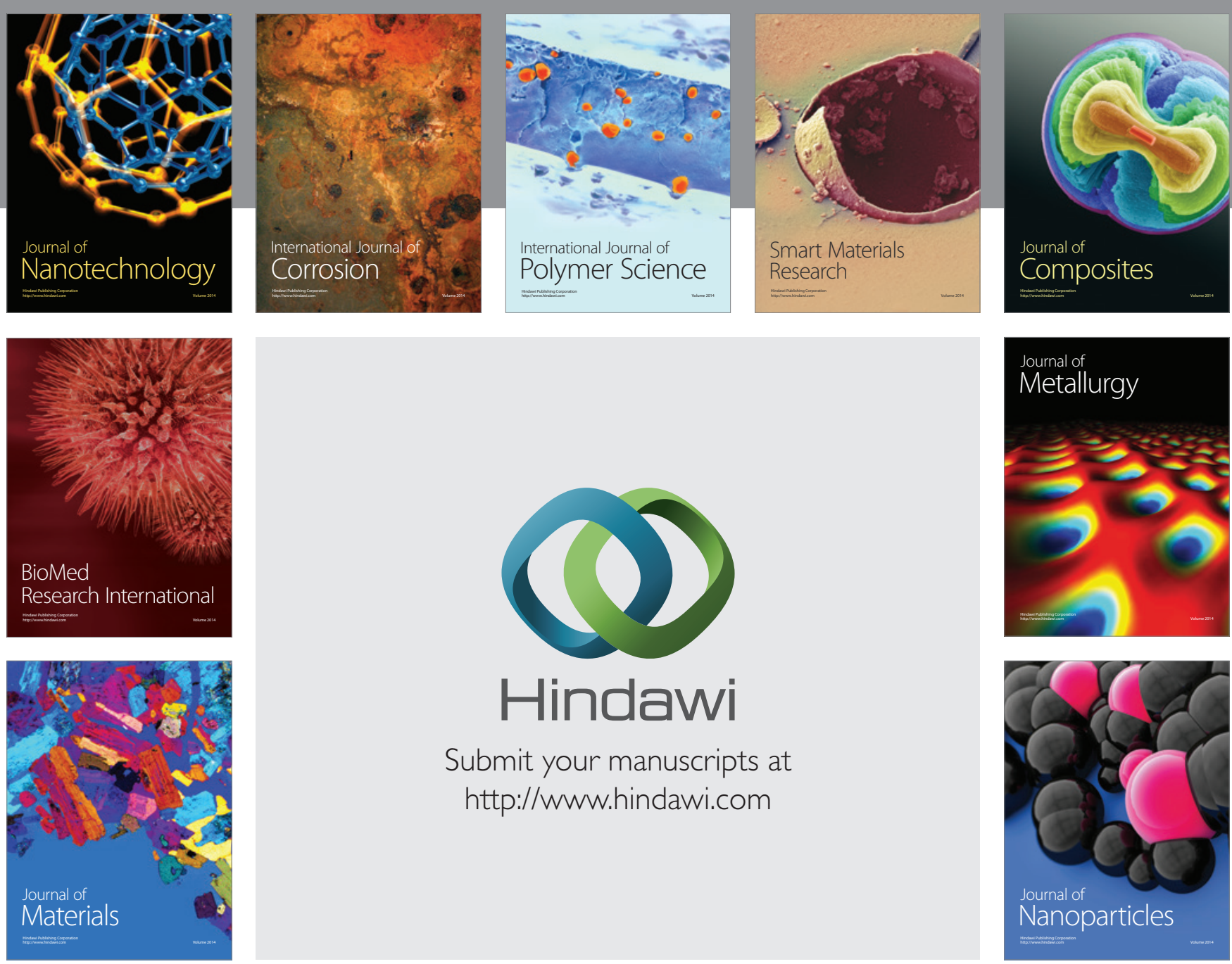

Submit your manuscripts at http://www.hindawi.com
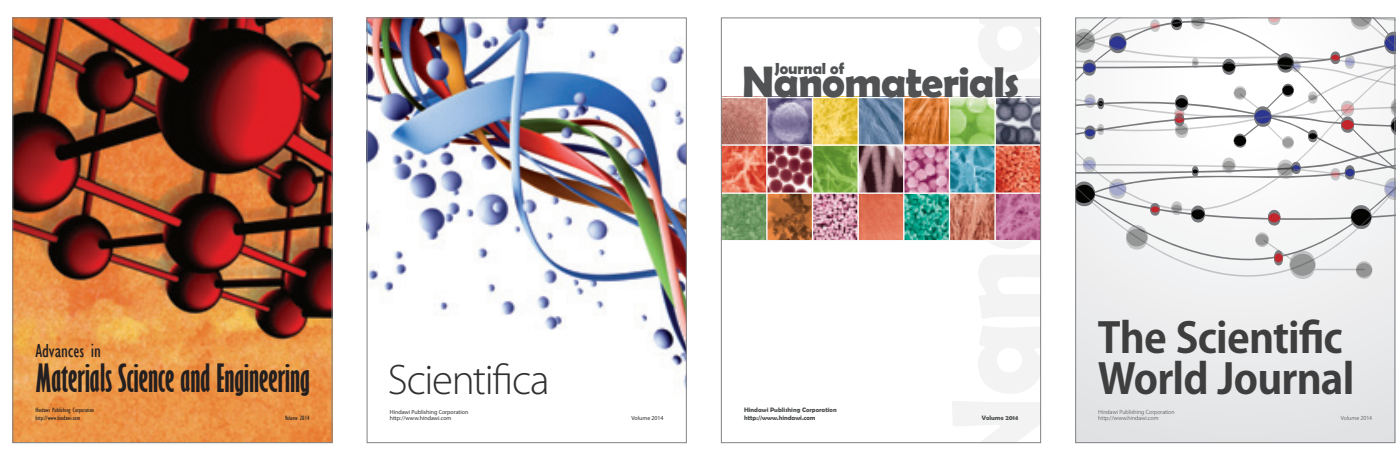

\section{The Scientific World Journal}
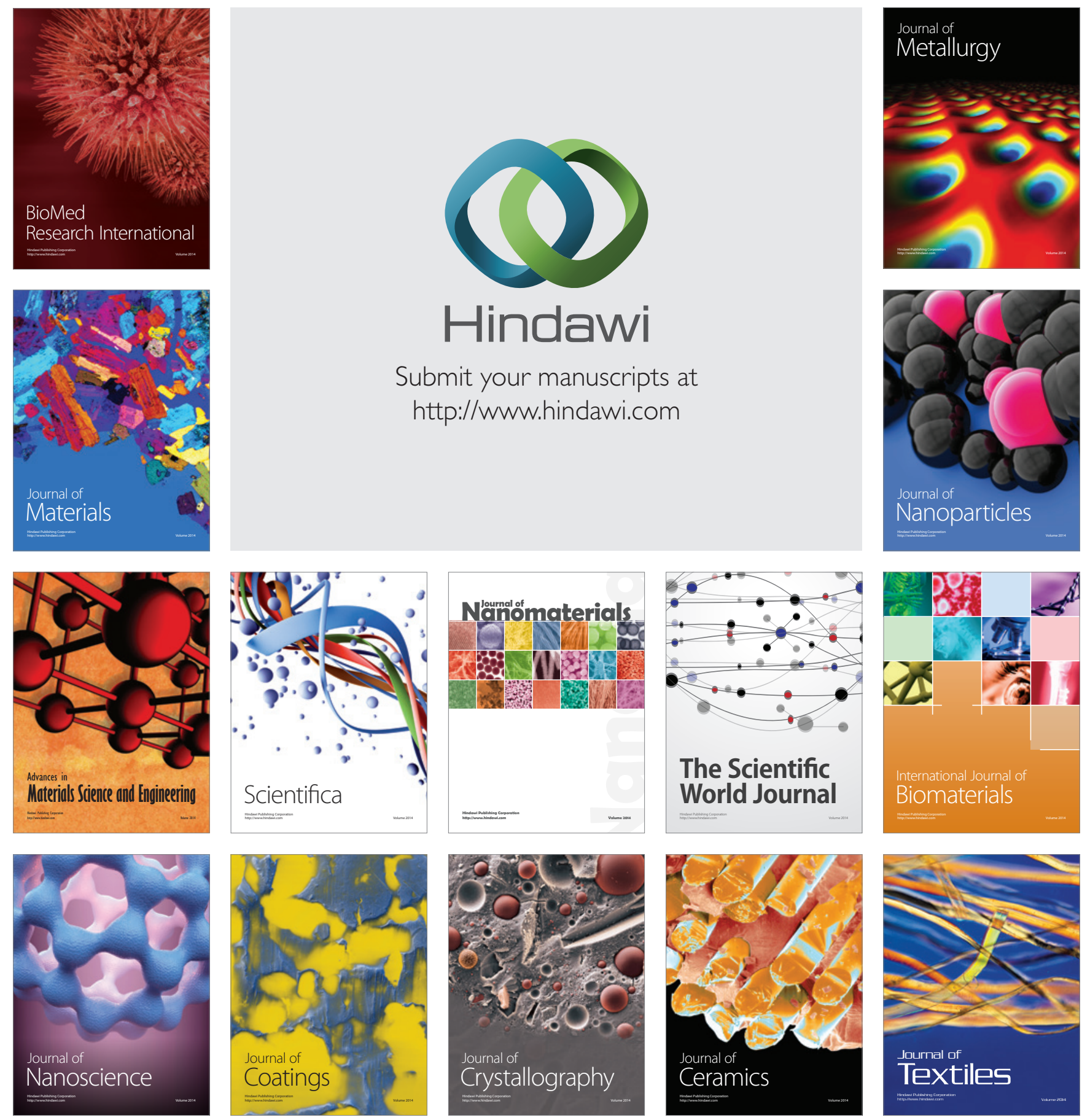